\title{
Effect of inorganic NPK fertilizers under different proportions on growth, yield and juice quality of sugarcane (Saccharum officinarum $\mathbf{L}$ ). \\ Abdul Fatah Soomro ${ }^{a^{*}}$, Shamasuddin Tunio ${ }^{\mathrm{b}}$, Mohammad Ibrahim Keerio ${ }^{\mathrm{b}}$,Inaitullah Rajper ${ }^{\mathrm{b}}$, Qammaruddin Chachar ${ }^{\mathrm{b}}$ and Mohammad Younis Arain ${ }^{\mathrm{c}}$ \\ ${ }^{a}$ Senior Scientific officer IPI, SARC, PARC Karachi, Pakistan \\ ${ }^{b}$ Professor Sindh Agriculture University, Tandojam, Pakistan \\ 'Principal Scientific Officer, SARC, PARC Karachi, Pakistan Corresponding author: fatahsoomro@ymail.com
}

\begin{abstract}
To determine the optimum NPK dose for maximum cane and sugar yield, the field experiment was conducted at Sugarcane Section, Agriculture Research Institute, Tandojam, Pakistan, located at $25^{\circ} 25^{\prime} 60^{\prime} \mathrm{N} 68^{\circ} 31^{\prime} 60 \mathrm{E}$ during 2008-09 and 2009-10. Sugarcane variety Thatta-10 was sown. The experiment, was meant to observe the effect of inorganic NPK fertilizers under different proportions on growth, yield and juice quality of sugarcane; comprised different inorganic NPK fertilizer doses viz. Control (0-0-0 NPK kg ha ${ }^{-1}$ ), $100 \%$ recommended (225-112-168 NPK $\mathrm{kg} \mathrm{ha}^{-1}$ ), 75\% less than recommended (56.25-28.0-42.0 NPK kg ha ${ }^{-1}$ ), 50\% less than recommended (112.5-56.0-84.0 NPK kg ha ${ }^{-1}$ ), 25\% less than recommended (168.75-84.0-126.0 NPK kg ha ${ }^{-1}$ ) and 25\% more than recommended (281.25-140.0-210.0 NPK kg ha ${ }^{-1}$ ). In the study agronomic, physiological, quality and nutrient uptake traits viz. tillers plant ${ }^{-1}$, plant height, stem girth, internodes plant ${ }^{-1}$, millable canes, cane yield, leaf area plant ${ }^{-1}$, leaf area index, crop growth rate, dry matter, brix, pol, purity, commercial cane sugar, NPK accumulation and uptake in plant and ratoon sugarcane crops were higher with the application of $100 \%$ recommended fertilizer at $225-112-168 \mathrm{NPK} \mathrm{kg}$ $\mathrm{ha}^{-1}$ and $25 \%$ more than recommended fertilizer at 281.25-140-210 NPK kg ha ${ }^{-1}$. The mean values of these both NPK levels were statistically non-significant. However, all inadequate applications beyond recommended applications significantly recorded less values of all traits of sugarcane plant and ratoon crop.
\end{abstract}

Key Words: Fertilizer, Sugarcane, Variety Thatta-10, Growth, Yield and Quality

\section{Introduction}

Sugarcane (Saccharum officinarum L.) has a great importance over the cash crops of Pakistan. The country is the world's fifth largest producer of Sugar in acreage and $15^{\text {th }}$ largest producer of Sugar [1] . The sugar industry is the country's second largest agroindustry after textile.

In addition to sugar, cane is employed in the production of alcohol for pharmaceuticals ethanol for fuel, baggase in paper and chip board manufacturing and press mud used as a rich source of organic matter and nutrients for crop production [2]. There are many reasons for low yields of sugarcane and low sugar recovery. Thus improved yield per acre may be achieved by the introduction of new high yield varieties of sugarcane.

Use of NPK fertilizers play key role in development of cane and sugar yields, but imbalanced fertilizer use seems to be one of the factors responsible for the constantly low cane yield in Pakistan. The proper fertilization is an important management tool for a healthy sugarcane crop to enhance sugarcane production significantly.

Sugarcane is known as a heavy feeder crop that depletes the soil of essential nutrients and therefore, adequate nutrient addition is important [3]. Nitrogen constitutes only a fraction of one per cent of the total dry matter of a mature sugarcane plant, as it plays a role as important as $\mathrm{C}, \mathrm{H}$ and $\mathrm{O}$, which together, form more than 90 percent of the dry matter. Sugarcane stores a higher percent of sucrose when nitrogen is limited for 6 to 8 weeks prior to harvest [4].

Proper fertilization is an important management function in sugarcane production [5]. Thus, it is necessary to supply sugarcane crop with the big three $(\mathrm{N}, \mathrm{P}$ and $\mathrm{K}$ ) to secure good cane quantity and quality. The chemical source of fertilizer (NPK) at the rate of $225-112-168 \mathrm{~kg} \mathrm{ha}^{-1}$ proved to be more effective to produce significantly greater plant height and thicker cane girth, more tillers, better brix, higher sugar recovery and maximum cane yield ha ${ }^{-1}$ [6]. Significant differences among fertilizer treatments like $200 \mathrm{~N} \mathrm{~kg} \mathrm{ha}^{-1}: 120 \mathrm{P}_{2} \mathrm{O}_{5} \mathrm{~kg}$ $\mathrm{ha}^{-1}: 150 \mathrm{~K}_{2} \mathrm{O} \mathrm{kg} \mathrm{ha}{ }^{-1}$ have been reported [5] .

There is no consistent statistically significant interaction shown to exist between $\mathrm{N}$ and $\mathrm{K}$ fertilization; however, inputs of $\mathrm{N}$ and $\mathrm{K}$ must be balanced to optimize sugarcane production [7].For high yield and good juice quality, $\mathrm{K}$ fertilizers are required in amounts equal to or greater than $\mathrm{N}$ and P. In most sugarcane producing countries of the world, NPK ratios of $2: 1: 3$ or $2: 1: 2$ or $3: 1: 5$ are commonly used [8]. N strongly stimulates growth, expansion of sugarcane canopy and interception of solar radiation [7] to primarily produce more mill able cane, a large amount of $\mathrm{K}$ is needed as an osmotic solute to maintain the necessary cell turgor to drive this $\mathrm{N}$-stimulated growth [8]. Applying $\mathrm{N}$ alone, NP, NK and NPK increased cane yields by $92.9,96.4,99.2$ and $105 \%$ 
respectively [9], cane yield was only 30-34 $\mathrm{t} \mathrm{ha}^{-1}$ with only $\mathrm{N}$ at $250 \mathrm{~kg} \mathrm{ha}^{-1}$, application of NPK with $\mathrm{K}$ at $125 \mathrm{~kg} \mathrm{~K} \mathrm{ha}^{-1}$ raised cane yield to $130-136 \mathrm{t} \mathrm{ha}^{-1}$ [10]. In Pakistan the average yield of the sugarcane varieties is much lower than their potential yield. For instance, an application of balanced NPK fertilizers, the potential yields are obtained up to $165.176 \mathrm{t} \mathrm{ha}^{-1}$ $[11,12,13]$ estimated potential cane yields of 150$200 \mathrm{t} \mathrm{ha}^{-1}$ for Sindh, 100-150 $\mathrm{t} \mathrm{ha}^{-1}$ for Punjab and 75$100 \mathrm{t} \mathrm{ha}^{-1}$ for NWFP. However, imbalanced fertilizer use seems to be one of the factors responsible for the constantly low cane yield in the country. [14] reported that fertilizer use for sugarcane cultivation in Pakistan is imbalanced and inappropriated. According to a survey report, only $4 \%$ of the cane growers use NPK and the majority $(73 \%)$ of them relies on NP fertilization. Fertilizers play an important role in increasing sugar production mainly because of their influence on cane tonnage. Most cane growers use fertilizers regularly to maintain or gain further increase in cane yields per acre. [15].

Yield potentiality of a crop would not reach a maximum unless proper fertilizer management is made. Sugarcane variety shows a tendency to decline in yield and vigor which needs replacement of the existing varieties with the new ones. Different sugarcane varieties differ in nutrient requirement from place to place according to soil and agroclimatic conditions [16, 17]. Some varieties have ability to absorb and utilize more nutrients from a soil under the same climatic condition and produce more cane and sugar. The application of NPK beyond 100 per cent of the recommended amount had produced only marginal increase in cane and sugar yield [18]. Sugar yield per unit area can be increased only, if there is simultaneous increase in the production of sugarcane and the recovery of sugar [19].Balanced application of nutrients (NPK) is the key factor to influence sugarcane production in Pakistan [20]. Moreover, due to excessive use of chemical fertilizers and absence of manuring, the soils have become most deficient in organic matter and other micronutrients. Besides, the farmers do not have more options for improvement of soil fertility.

Keeping in view the importance of balanced use of chemical fertilizers a new sugarcane variety Thatta 10 was tested at different proportions of NPK fertilizers to achieve the maximum cane and sugar recovery. New sugarcane variety Thatta-10 is progeny of local variety L-113 developed by National Sugar Crops Research Institute (NSCRI), PARC, Thatta was approved on 16th Oct. 2004. The variety has potential of producing 200+Tons/ha cane yield and sugar recovery of $14 \%$.The variety is drought tolerant, resistant to lodging and provide very good ratoon crop.

\section{Materials and Methods}

The experiment was conducted at Sugarcane Section, Agriculture Research Institute, Tandojam, Pakistan, located at $25^{\circ} 25.19 \mathrm{~N} 68^{\circ} 32.07 \mathrm{E}$. The soil used in study was soltanpur series (Aridisols) with clay loam in texture, non saline, EC $\left(0.96 \mathrm{dS} \mathrm{m}^{-1}\right)$, slightly alkaline $\mathrm{pH}$ (7.8), calcareous $\left(\mathrm{CaCO}_{3} 9.8 \%\right)$, low in organic matter $(0.8 \%)$, total nitrogen content $(0.04 \%)$ and available phosphorus $\left(3.8 \mathrm{mg} \mathrm{kg}^{-1}\right)$, however high in exchangeable potassium $\left(170 \mathrm{mg} \mathrm{kg}^{-1}\right)$.

The experiment was designed under randomized complete block design with three replications having the plot size of 7 × $5 \mathrm{~m}=35 \mathrm{~m}^{2}$. The treatments applied to plant and ratoon sugarcane crops are as under:

\begin{tabular}{llll}
\multicolumn{1}{c}{ TREATMENT } & N & P & K \\
$\mathrm{T}_{1=}$ Control (untreated) & 0 & 0 & 0 \\
$\mathrm{~T}_{2=1} 00 \%$ recommended dose of & 225.0 & 112.0 & 168.0 \\
$\mathrm{NPK}$ & & & \\
$\mathrm{T}_{3=} 75 \%$ less than recommended & 56.25 & 28.0 & 42.0 \\
$\mathrm{NPK}$ & & & \\
$\mathrm{T}_{4=50 \%}$ less than recommended & 112.5 & 56.0 & 84.0 \\
$\mathrm{NPK}$ & & & \\
$\mathrm{T}_{5=25 \% \text { less than recommended }}$ & 168.75 & 84.0 & 126.0 \\
$\mathrm{NPK}$ & & & \\
$\mathrm{T}_{6==}$ 25\% more than & & & \\
recommended NPK & 281.25 & 140.0 & 210.0
\end{tabular}

\section{Cultural practices Seedbed preparation}

The land was initially ploughed to eliminate the hard pan with disc plow. The soil was left to dry for 15 days. After crushing the clods, dry weeds and stubbles were removed. The precise land leveling was done to achieve fine seedbed, followed disc harrow and rotavator used to complete the seedbed preparation. A ridger was used to develop ridges at $100 \mathrm{~cm}$ distance.

Planting time and method

During the $2^{\text {nd }}$ week of September the crop was planted manually and the sets bearing 2-3 buds of sugarcane variety Thatta-10 were placed in the furrows (end to end) and covered with thin soil layer . 
Table- 01. Agronomic traits of sugarcane plant and ratoon crop as affected by inorganic NPK levels.

\begin{tabular}{|c|c|c|c|c|c|c|c|c|c|c|c|c|}
\hline \multirow[t]{2}{*}{ Treatments } & \multicolumn{2}{|c|}{ Tillers Plant $^{-1}$} & \multicolumn{2}{|c|}{$\begin{array}{l}\text { Plant height } \\
(\mathrm{cm})\end{array}$} & \multicolumn{2}{|c|}{$\begin{array}{l}\text { Stem girth } \\
(\mathrm{cm})\end{array}$} & \multicolumn{2}{|c|}{$\begin{array}{l}\text { Internodes } \\
\text { plant }^{-1}\end{array}$} & \multicolumn{2}{|c|}{$\begin{array}{l}\text { Millable canes } \\
\left(000 \mathrm{ha}^{-1}\right)\end{array}$} & \multicolumn{2}{|c|}{$\begin{array}{l}\text { Cane yield } \\
\left(\mathrm{t} \mathrm{ha}^{-1}\right)\end{array}$} \\
\hline & $\begin{array}{l}\text { Plant } \\
\text { Crop }\end{array}$ & $\begin{array}{l}\text { Ratoon } \\
\text { Crop }\end{array}$ & $\begin{array}{l}\text { Plant } \\
\text { Crop }\end{array}$ & $\begin{array}{l}\text { Ratoon } \\
\text { Crop }\end{array}$ & $\begin{array}{l}\text { Plant } \\
\text { Crop }\end{array}$ & $\begin{array}{l}\text { Ratoon } \\
\text { Crop }\end{array}$ & $\begin{array}{l}\text { Plant } \\
\text { Crop }\end{array}$ & $\begin{array}{l}\text { Ratoon } \\
\text { Crop }\end{array}$ & $\begin{array}{l}\text { Plant } \\
\text { Crop }\end{array}$ & $\begin{array}{l}\text { Ratoon } \\
\text { Crop }\end{array}$ & $\begin{array}{l}\text { Plant } \\
\text { Crop }\end{array}$ & $\begin{array}{l}\text { Ratoon } \\
\text { Crop }\end{array}$ \\
\hline $\begin{array}{l}\text { Control (untreated) } \\
0-0-0 \text { NPK kg ha }\end{array}$ & $3.6 \mathrm{c}$ & $4.2 \mathrm{~d}$ & $212.8 \mathrm{c}$ & $206.6 \mathrm{~b}$ & $2.36 \mathrm{~d}$ & $2.23 \mathrm{~d}$ & $\begin{array}{c}18.77 \\
b\end{array}$ & $18.11 \mathrm{c}$ & $\begin{array}{c}43.30 \\
\mathrm{~d}\end{array}$ & $42.83 \mathrm{c}$ & $\begin{array}{c}33.00 \\
\mathrm{~d}\end{array}$ & $31.00 \mathrm{~d}$ \\
\hline $\begin{array}{l}100 \% \text { Recommended }\left(225-112-168^{\text {NPK kg ha }}{ }^{-1}\right)\end{array}$ & $5.1 \mathrm{a}$ & $5.5 \mathrm{ab}$ & $246.8 \mathrm{a}$ & $236.6 \mathrm{a}$ & $2.54 \mathrm{a}$ & $2.53 \mathrm{a}$ & $\begin{array}{c}21.88 \\
\mathrm{a}\end{array}$ & $21.55 \mathrm{a}$ & $\begin{array}{c}95.89 \\
\mathrm{a}\end{array}$ & $95.96 \mathrm{a}$ & $\begin{array}{c}96.00 \\
\mathrm{a}\end{array}$ & $96.65 \mathrm{a}$ \\
\hline $\begin{array}{l}75 \% \text { less than recommended }(56.25- \\
28.0-42.0 \mathrm{NPK} \mathrm{kg} \mathrm{ha} \\
-1\end{array}$ & $3.9 \mathrm{bc}$ & $4.3 \mathrm{~cd}$ & $223.3 \mathrm{~b}$ & $207.3 \mathrm{~b}$ & $\begin{array}{c}2.42 \\
\mathrm{~cd}\end{array}$ & $2.29 \mathrm{~cd}$ & $\begin{array}{c}19.66 \\
b\end{array}$ & $18.77 \mathrm{c}$ & $\begin{array}{c}69.50 \\
\mathrm{c}\end{array}$ & $67.33 \mathrm{~b}$ & $\begin{array}{c}57.00 \\
\mathrm{c}\end{array}$ & $57.66 \mathrm{c}$ \\
\hline $\begin{array}{l}50 \% \text { less than recommended }(112.5- \\
\left.56.0-84.0 \mathrm{NPK} \mathrm{kg} \mathrm{h}^{-1}\right)\end{array}$ & $4.5 \mathrm{abc}$ & $4.7 \mathrm{bcd}$ & $231.1 \mathrm{~b}$ & $227.0 \mathrm{a}$ & $\begin{array}{l}2.43 \\
\text { bcd }\end{array}$ & $2.32 \mathrm{c}$ & $\begin{array}{c}19.32 \\
b\end{array}$ & $19.22 \mathrm{bc}$ & $\begin{array}{c}74.72 \\
\mathrm{bc}\end{array}$ & $\begin{array}{c}77.73 \\
\mathrm{ab}\end{array}$ & $\begin{array}{c}66.33 \\
b c\end{array}$ & $\begin{array}{c}67.00 \\
b c\end{array}$ \\
\hline $\begin{array}{l}25 \% \text { less than recommended (168.75- } \\
\left.84.0-126.0 \mathrm{NPK} \mathrm{kg} \mathrm{ha}^{-1}\right)\end{array}$ & $4.7 \mathrm{ab}$ & $5.1 \mathrm{abc}$ & $232.6 \mathrm{~b}$ & $230.3 \mathrm{a}$ & $\begin{array}{c}2.45 \\
b c\end{array}$ & $2.43 \mathrm{~b}$ & $\begin{array}{c}20.22 \\
b\end{array}$ & $20.44 \mathrm{ab}$ & $\begin{array}{c}83.62 \\
a b\end{array}$ & $\begin{array}{c}80.00 \\
a b\end{array}$ & $\begin{array}{c}75.00 \\
b\end{array}$ & $76.00 \mathrm{~b}$ \\
\hline $\begin{array}{l}25 \% \text { more than recommended (281.25- } \\
\left.140.0-210.0 \mathrm{NPK} \mathrm{kg} \mathrm{ha}^{-1}\right)\end{array}$ & $5.3 \mathrm{a}$ & $5.7 \mathrm{a}$ & $244.1 \mathrm{a}$ & $234.6 \mathrm{a}$ & $2.55 \mathrm{a}$ & $2.51 \mathrm{ab}$ & $\begin{array}{c}21.88 \\
\mathrm{a}\end{array}$ & $21.66 \mathrm{a}$ & $\begin{array}{c}96.17 \\
\mathrm{a}\end{array}$ & $94.26 \mathrm{a}$ & $\begin{array}{c}94.70 \\
\mathrm{a}\end{array}$ & $95.06 \mathrm{a}$ \\
\hline S.E & 0.42 & 0.36 & 4.24 & 5.76 & 0.32 & 0.03 & 0.73 & 0.68 & 5.84 & 8.46 & 7.96 & 7.06 \\
\hline LSD (5\%) & 0.95 & 0.80 & 9.44 & 12.85 & 0.73 & 0.07 & 1.63 & 1.52 & 13.02 & 18.86 & 17.74 & 15.73 \\
\hline
\end{tabular}

In each column, means followed by common letter are not significantly different at $5 \%$ probability level. 
Table-02. Physiological parameters of sugarcane plant and ratoon crop as affected by inorganic NPK levels.

\begin{tabular}{|c|c|c|c|c|c|c|c|c|}
\hline \multirow[t]{2}{*}{ Treatments } & \multicolumn{2}{|c|}{$\begin{array}{l}\text { Leaf area plant }{ }^{-1} \\
\left(\mathrm{~cm}^{2}\right)\end{array}$} & \multicolumn{2}{|c|}{ Leaf area index } & \multicolumn{2}{|c|}{$\begin{array}{l}\text { Crop growth rate } \\
\left(\mathrm{gm}^{2} \text { day }^{-1}\right)\end{array}$} & \multicolumn{2}{|c|}{$\begin{array}{l}\text { Dry matter } \\
\left(\mathrm{g} \mathrm{m}^{-2}\right)\end{array}$} \\
\hline & $\begin{array}{l}\text { Plant } \\
\text { Crop }\end{array}$ & $\begin{array}{l}\text { Ratoon } \\
\text { Crop }\end{array}$ & $\begin{array}{l}\text { Plant } \\
\text { Crop }\end{array}$ & $\begin{array}{l}\text { Ratoon } \\
\text { Crop }\end{array}$ & $\begin{array}{l}\text { Plant } \\
\text { Crop }\end{array}$ & $\begin{array}{l}\text { Ratoon } \\
\text { Crop }\end{array}$ & $\begin{array}{l}\text { Plant } \\
\text { Crop } \\
\end{array}$ & $\begin{array}{l}\text { Ratoon } \\
\text { Crop }\end{array}$ \\
\hline Control (untreated) 0-0-0 NPK kg ha ${ }^{-1}$ & $3000 \mathrm{~d}$ & $2917 \mathrm{~d}$ & $5.40 \mathrm{~d}$ & $5.25 \mathrm{~d}$ & $5.33 \mathrm{~d}$ & $5.63 \mathrm{~d}$ & $3200 \mathrm{~d}$ & $3017 \mathrm{~d}$ \\
\hline $\begin{array}{l}100 \% \text { Recommended (225 -112-168 } \\
\text { NPK kg ha-1) }\end{array}$ & $5400 \mathrm{a}$ & 5643 a & $9.72 \mathrm{a}$ & $10.16 \mathrm{a}$ & $8.33 \mathrm{a}$ & $9.75 \mathrm{a}$ & $5000 \mathrm{a}$ & $5225 \mathrm{a}$ \\
\hline $\begin{array}{l}50 \% \text { less than recommended }(112.5-56.0- \\
\left.84.0 \mathrm{NPK} \mathrm{kg} \mathrm{ha}^{-1}\right)\end{array}$ & $3600 \mathrm{c}$ & $3762 \mathrm{c}$ & $6.48 c$ & $6.77 \mathrm{c}$ & $6.16 \mathrm{bc}$ & $7.22 \mathrm{bc}$ & $3700 \mathrm{bc}$ & $3867 \mathrm{bc}$ \\
\hline $\begin{array}{l}25 \% \text { less than recommended }(168.75- \\
84.0-126.0 \mathrm{NPK} \mathrm{kg} \mathrm{ha-1})\end{array}$ & $4200 \mathrm{~b}$ & $4389 \mathrm{~b}$ & $7.56 \mathrm{~b}$ & $7.90 \mathrm{~b}$ & $6.66 \mathrm{~b}$ & $7.80 \mathrm{~b}$ & $4000 \mathrm{~b}$ & $4180 \mathrm{~b}$ \\
\hline S.E & 130.0 & 135.0 & 0.23 & 0.24 & 0.23 & 0.27 & 142.2 & 147.0 \\
\hline $\operatorname{LSD}(5 \%)$ & 409.5 & 425.4 & 0.73 & 0.76 & 0.74 & 0.86 & 448.1 & 463.1 \\
\hline
\end{tabular}

In each column, means followed by common letter are not significantly different at 5\% probability level. 
Table-03. Qualitative traits of sugarcane plant and ratoon crop as affected by different NPK levels.

\begin{tabular}{|c|c|c|c|c|c|c|c|c|c|c|}
\hline \multirow[t]{2}{*}{ Treatments } & \multicolumn{2}{|c|}{$\begin{array}{l}\text { Fiber } \\
(\%)\end{array}$} & \multicolumn{2}{|c|}{$\begin{array}{l}\text { Brix } \\
(\%)\end{array}$} & \multicolumn{2}{|c|}{$\begin{array}{l}\text { Pol } \\
(\%)\end{array}$} & \multicolumn{2}{|c|}{$\begin{array}{l}\text { Purity } \\
(\%)\end{array}$} & \multicolumn{2}{|c|}{$\begin{array}{c}\text { Commercial } \\
\text { Cane sugar }(\%)\end{array}$} \\
\hline & $\begin{array}{l}\text { Plant } \\
\text { Crop }\end{array}$ & $\begin{array}{l}\text { Ratoon } \\
\text { Crop } \\
\end{array}$ & $\begin{array}{l}\text { Plant } \\
\text { Crop }\end{array}$ & $\begin{array}{l}\text { Ratoon } \\
\text { Crop }\end{array}$ & $\begin{array}{l}\text { Plant } \\
\text { Crop }\end{array}$ & $\begin{array}{l}\text { Ratoon } \\
\text { Crop }\end{array}$ & $\begin{array}{l}\text { Plant } \\
\text { Crop }\end{array}$ & $\begin{array}{l}\text { Ratoon } \\
\text { Crop }\end{array}$ & $\begin{array}{l}\text { Plant } \\
\text { Crop }\end{array}$ & $\begin{array}{l}\text { Ratoon } \\
\text { Crop }\end{array}$ \\
\hline Control (untreated) $0-0-0$ NPK kg ha & $\begin{array}{c}12.28 \\
\mathrm{a}\end{array}$ & $12.29 \mathrm{a}$ & $\begin{array}{c}20.63 \\
d\end{array}$ & $20.80 \mathrm{e}$ & $\begin{array}{c}15.67 \\
\mathrm{f}\end{array}$ & $15.87 \mathrm{e}$ & $75.95 \mathrm{e}$ & $76.03 \mathrm{f}$ & $10.52 \mathrm{f}$ & $10.70 \mathrm{f}$ \\
\hline $\begin{array}{l}100 \% \text { Recommended }(225-112-168 \\
\text { NPK kg ha }\end{array}$ & $\begin{array}{c}12.18 \\
\mathrm{~d}\end{array}$ & $12.16 \mathrm{e}$ & $\begin{array}{c}22.63 \\
\mathrm{a}\end{array}$ & $23.16 \mathrm{a}$ & $\begin{array}{c}18.92 \\
b\end{array}$ & $19.22 \mathrm{a}$ & $83.60 \mathrm{a}$ & $82.90 \mathrm{~b}$ & $13.66 \mathrm{~b}$ & $13.79 \mathrm{~b}$ \\
\hline $\begin{array}{l}75 \% \text { less than recommended }(56.25- \\
\left.28.0-42.0 \mathrm{NPK} \mathrm{kg} \mathrm{ha}{ }^{-1}\right)\end{array}$ & $\begin{array}{c}12.27 \\
\mathrm{ab}\end{array}$ & $12.27 \mathrm{~b}$ & $\begin{array}{c}20.80 \\
\mathrm{~d}\end{array}$ & $21.10 \mathrm{~d}$ & $\begin{array}{c}15.94 \\
\mathrm{e}\end{array}$ & $16.20 \mathrm{~d}$ & $76.62 \mathrm{~d}$ & $76.77 \mathrm{e}$ & $10.78 \mathrm{e}$ & $10.97 \mathrm{e}$ \\
\hline $\begin{array}{l}50 \% \text { less than recommended (112.5- } \\
\left.56.0-84.0 \mathrm{NPK} \mathrm{kg} \mathrm{ha}{ }^{-1}\right)\end{array}$ & $\begin{array}{c}12.23 \\
\mathrm{bc}\end{array}$ & $12.22 \mathrm{c}$ & $\begin{array}{c}21.53 \\
\mathrm{c}\end{array}$ & $21.83 \mathrm{c}$ & $\begin{array}{c}16.68 \\
d\end{array}$ & $16.90 \mathrm{c}$ & $77.45 \mathrm{c}$ & $77.43 \mathrm{~d}$ & $11.37 \mathrm{~d}$ & $11.52 \mathrm{~d}$ \\
\hline $\begin{array}{l}25 \% \text { less than recommended }(168.75- \\
\left.84.0-126.0 \mathrm{NPK} \mathrm{kg} \mathrm{ha}{ }^{-1}\right)\end{array}$ & $\begin{array}{c}12.21 \\
\mathrm{~cd}\end{array}$ & $12.19 \mathrm{~d}$ & $\begin{array}{c}22.36 \\
b\end{array}$ & $22.66 \mathrm{~b}$ & $\begin{array}{l}17.68 \\
\mathrm{c}\end{array}$ & $17.95 \mathrm{~b}$ & $79.04 \mathrm{~b}$ & $79.17 \mathrm{c}$ & $12.25 \mathrm{c}$ & $12.45 \mathrm{c}$ \\
\hline $\begin{array}{l}25 \% \text { more than recommended } \\
\left(281.25-140.0-210.0 \mathrm{NPK} \mathrm{kg} \mathrm{ha}{ }^{-1}\right)\end{array}$ & $\begin{array}{c}12.19 \\
\mathrm{~d}\end{array}$ & $12.17 \mathrm{e}$ & $\begin{array}{c}22.83 \\
\mathrm{a}\end{array}$ & $23.16 \mathrm{a}$ & $\begin{array}{c}19.09 \\
\mathrm{a}\end{array}$ & $19.37 \mathrm{a}$ & $83.61 \mathrm{a}$ & $83.67 \mathrm{a}$ & $13.77 \mathrm{a}$ & $13.98 \mathrm{a}$ \\
\hline S.E & 0.01 & 6.26 & 0.11 & 0.09 & 0.62 & 0.07 & 0.19 & 0.25 & 0.04 & 0.06 \\
\hline LSD (5\%) & 0.03 & 0.01 & 0.26 & 0.20 & 0.13 & 0.15 & 0.44 & 0.56 & 0.08 & 0.13 \\
\hline
\end{tabular}

In each column, means followed by common letter are not significantly different at 5\% probability level. 
Table-04. NPK concentration and uptake in sugarcane plant and ratoon crop as affected by inorganic NPK levels.

\begin{tabular}{|c|c|c|c|c|c|c|c|c|c|c|c|c|}
\hline \multirow[t]{2}{*}{ Treatments } & \multicolumn{2}{|l|}{$\begin{array}{l}\mathrm{N} \\
(\%)\end{array}$} & \multicolumn{2}{|l|}{$\begin{array}{l}\mathrm{P} \\
(\%)\end{array}$} & \multicolumn{2}{|l|}{$\begin{array}{l}\mathrm{K} \\
(\%)\end{array}$} & \multicolumn{2}{|c|}{$\begin{array}{l}\mathrm{N} \\
\text { uptake } \\
\left(\mathrm{kg} \mathrm{ha}^{-1}\right)\end{array}$} & \multicolumn{2}{|c|}{$\begin{array}{l}\mathrm{P} \\
\text { uptake } \\
\left(\mathrm{kg} \mathrm{ha}^{-1}\right)\end{array}$} & \multicolumn{2}{|c|}{$\begin{array}{l}\mathrm{K} \\
\text { uptake } \\
\left(\mathrm{kg} \mathrm{ha}^{-1}\right)\end{array}$} \\
\hline & $\begin{array}{l}\text { Plant } \\
\text { Crop }\end{array}$ & $\begin{array}{l}\text { Ratoon } \\
\text { Crop }\end{array}$ & $\begin{array}{l}\text { Plant } \\
\text { Crop }\end{array}$ & $\begin{array}{l}\text { Ratoon } \\
\text { Crop }\end{array}$ & $\begin{array}{l}\text { Plant } \\
\text { Crop }\end{array}$ & $\begin{array}{l}\text { Ratoon } \\
\text { Crop }\end{array}$ & $\begin{array}{l}\text { Plant } \\
\text { Crop }\end{array}$ & $\begin{array}{l}\text { Ratoon } \\
\text { Crop }\end{array}$ & $\begin{array}{l}\text { Plant } \\
\text { Crop }\end{array}$ & $\begin{array}{l}\text { Ratoon } \\
\text { Crop }\end{array}$ & $\begin{array}{l}\text { Plant } \\
\text { Crop }\end{array}$ & $\begin{array}{l}\text { Ratoon } \\
\text { Crop }\end{array}$ \\
\hline $\begin{array}{l}\text { Control (untreated) } \\
0-0-0 \text { NPK kg ha }\end{array}$ & $1.14 \mathrm{c}$ & $1.12 \mathrm{e}$ & $0.19 \mathrm{c}$ & $0.18 \mathrm{~d}$ & $1.21 \mathrm{e}$ & $1.21 \mathrm{e}$ & $\begin{array}{c}61.23 \\
\mathrm{e}\end{array}$ & $\begin{array}{c}61.43 \\
\mathrm{e}\end{array}$ & $10.46 \mathrm{e}$ & $10.200 \mathrm{f}$ & $\begin{array}{c}64.67 \\
\mathrm{f}\end{array}$ & $\begin{array}{c}66.50 \\
f\end{array}$ \\
\hline $\begin{array}{l}100 \% \text { Recommended (225 -112- } \\
\left.168 \mathrm{NPK} \mathrm{kg} \mathrm{ha}^{-1}\right)\end{array}$ & $\begin{array}{c}1.35 \\
\mathrm{ab}\end{array}$ & $1.36 \mathrm{a}$ & $0.30 \mathrm{~b}$ & $0.37 \mathrm{a}$ & $\begin{array}{c}1.58 \\
\mathrm{ab}\end{array}$ & $1.62 \mathrm{a}$ & $\begin{array}{c}128.50 \\
\mathrm{a}\end{array}$ & $\begin{array}{c}129.77 \\
\mathrm{a}\end{array}$ & $28.40 \mathrm{~b}$ & $\begin{array}{c}35.200 \\
\mathrm{~b}\end{array}$ & $\begin{array}{c}150.37 \\
b\end{array}$ & $\begin{array}{c}154.67 \\
b\end{array}$ \\
\hline $\begin{array}{l}75 \% \text { less than recommended } \\
\left(56.25-28.0-42.0 \mathrm{NPK} \mathrm{kg} \mathrm{ha}^{-1}\right)\end{array}$ & $\begin{array}{c}1.25 \\
\mathrm{bc}\end{array}$ & $1.20 \mathrm{~d}$ & $0.25 \mathrm{~b}$ & $0.28 \mathrm{c}$ & $1.41 \mathrm{~d}$ & $1.37 \mathrm{~d}$ & $\begin{array}{c}71.60 \\
\mathrm{~d}\end{array}$ & $\begin{array}{c}72.63 \\
\mathrm{~d}\end{array}$ & $14.70 \mathrm{~d}$ & $\begin{array}{c}16.933 \\
\mathrm{e}\end{array}$ & $\begin{array}{c}80.77 \\
\mathrm{e}\end{array}$ & $\begin{array}{c}83.10 \\
\mathrm{e}\end{array}$ \\
\hline $\begin{array}{l}50 \% \text { less than recommended } \\
\left(112.5-56.0-84.0 \mathrm{NPK} \mathrm{kg} \mathrm{ha}^{-1}\right)\end{array}$ & $\begin{array}{c}1.27 \\
\mathrm{ab}\end{array}$ & $1.22 \mathrm{~cd}$ & $0.27 \mathrm{~b}$ & $0.29 \mathrm{bc}$ & $1.47 \mathrm{c}$ & $1.43 \mathrm{c}$ & $\begin{array}{c}84.97 \\
c\end{array}$ & $\begin{array}{c}82.30 \\
\mathrm{c}\end{array}$ & $\begin{array}{c}18.10 \\
\mathrm{~cd}\end{array}$ & $\begin{array}{c}19.833 \\
d\end{array}$ & $\begin{array}{c}97.93 \\
d\end{array}$ & $96.50 \mathrm{~d}$ \\
\hline $\begin{array}{l}25 \% \text { less than recommended } \\
\left(168.75-84.0-126.0 \mathrm{NPK} \mathrm{kg} \mathrm{ha}^{-1}\right)\end{array}$ & $\begin{array}{c}1.27 \\
\mathrm{ab}\end{array}$ & $1.28 \mathrm{bc}$ & $0.28 \mathrm{~b}$ & $0.31 \mathrm{~b}$ & $1.53 \mathrm{~b}$ & $1.52 \mathrm{~b}$ & $\begin{array}{c}96.57 \\
b\end{array}$ & $\begin{array}{c}97.43 \\
b\end{array}$ & $21.73 \mathrm{c}$ & $\begin{array}{c}23.933 \\
c\end{array}$ & $\begin{array}{c}116.07 \\
\mathrm{c}\end{array}$ & $\begin{array}{c}115.83 \\
\mathrm{c}\end{array}$ \\
\hline $\begin{array}{l}25 \% \text { more than recommended } \\
\left(281.25-140.0-210.0 \mathrm{NPK} \mathrm{kg} \mathrm{ha}^{-1}\right)\end{array}$ & $1.36 \mathrm{a}$ & $1.35 \mathrm{ab}$ & $0.37 \mathrm{a}$ & $0.39 \mathrm{a}$ & $1.63 \mathrm{a}$ & $1.64 \mathrm{a}$ & $\begin{array}{c}131.50 \\
\mathrm{a}\end{array}$ & $\begin{array}{c}131.30 \\
\mathrm{a}\end{array}$ & $35.70 \mathrm{a}$ & $\begin{array}{c}38.233 \\
\mathrm{a}\end{array}$ & $\begin{array}{c}157.27 \\
\mathrm{a}\end{array}$ & $\begin{array}{c}160.17 \\
\mathrm{a}\end{array}$ \\
\hline S.E & 0.05 & 0.315 & 0.02 & 0.135 & 0.02 & 0.023 & 3.44 & 2.161 & 1.83 & 0.9607 & 2.43 & 1.3653 \\
\hline $\operatorname{LSD}(5 \%)$ & 0.11 & 0.70 & 0.04 & 0.03 & 0.54 & 0.05 & 7.67 & 4.81 & 4.07 & 2.14 & 5.41 & 3.04 \\
\hline
\end{tabular}

In each column, means followed by common letter are not significantly different at $5 \%$ probability level. 


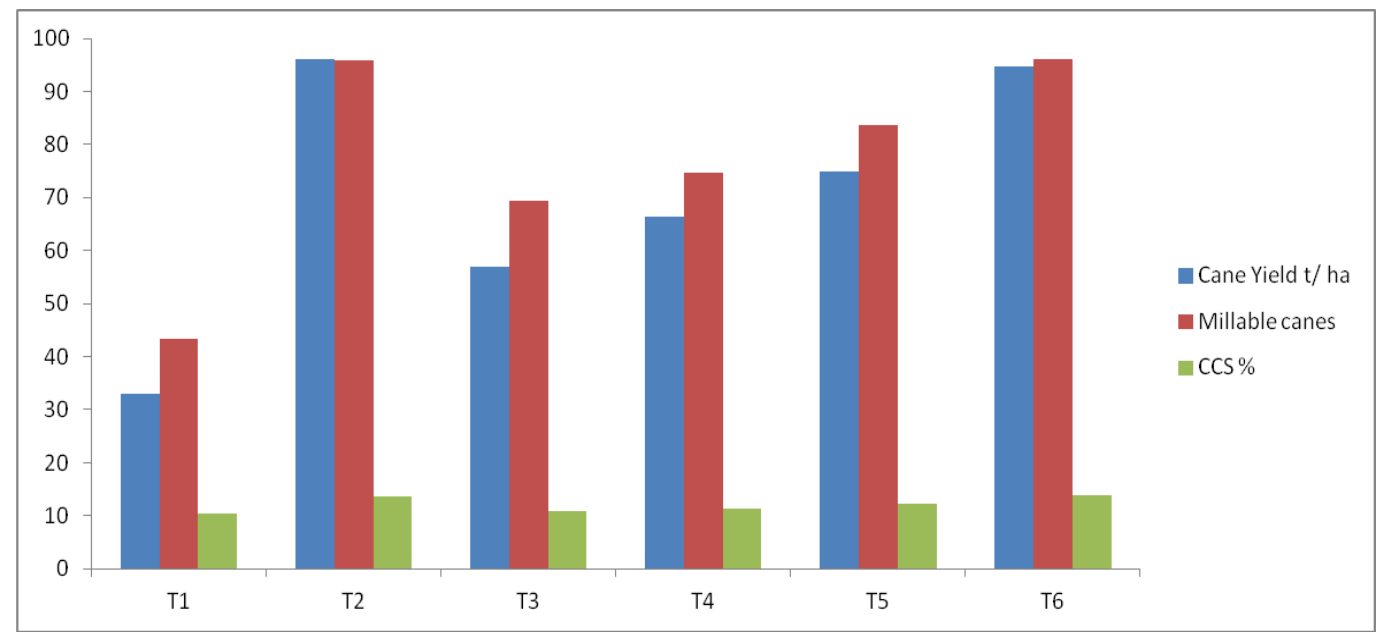

Figure 1: Effect of inorganic Fertilizer levels on quantitative and qualitative traits of Sugarcane crop

\section{Irrigation}

After completion of the sowing process, a light irrigation was applied in furrows. Consequently, the irrigation water was applied at 15 days interval in winter and at 8-10 days interval in summer months. Overall, twenty five irrigations were applied during the growing season.

\section{Fertilizer}

Nitrogen, phosphorous and potassium were applied according to the experimental treatments in the form of Urea, DAP and SOP. Nitrogen was split applied in three equal doses i.e. at sowing, after germination and at tillering. All phosphorus and potash were applied at sowing in plant cane. However, in ratoon crop, Phosphorus and potassium were applied after stubble shaving, followed by inter-row cultivation; whereas nitrogen was split applied during $1^{\text {st }}$ and $4^{\text {th }}$ irrigations, and after completion of tillering.

\section{After care}

Two earthingup (putting soil on root zone) were carried out in plant and ratoon crops. The interculturing was done to control the weeds and stem borer infestation was controlled through, biological control measures by releasing Trichogramma chilonis (Lshii) in the fields. For combating the termites, Lorsban was applied at the rate of $5 \mathrm{~L} \mathrm{ha}^{-1}$ with $1^{\text {st }}$ irrigation.

\section{Results and Discussions}

\section{Effect of inorganic NPK on agronomic traits of} plant and ratoon crop

The analysis of variance for all agronomic traits of sugarcane plant and ratoon crop as affected by inorganic NPK levels showed significant difference at $5 \%$ probability level except germination which was non-significant. The results of the study revealed maximum tillers plant ${ }^{-1}(5.1-5.3),(5.5-5.7)$, plant height $(244.1-246.8 \mathrm{~cm})$, (234.6-236.6) ,stem girth $(2.54-2.55 \mathrm{~cm}),(2.51-2.53 \mathrm{~cm})$, internodes plant ${ }^{-1}$ (21.88), (21.55-21.66), millable canes (95.8-96.1 thousands $\left.\mathrm{ha}^{-1}\right),\left(94.2-95.9\right.$ thousands $\left.\mathrm{ha}^{-1}\right)$ and cane yield (94.7-96.0 $\left.\mathrm{t} \mathrm{ha}^{-1}\right)$, (95.0-96.6 $\left.\mathrm{t} \mathrm{ha}^{-1}\right)$ was recorded from plant and ratoon crop, respectively with the application of $100 \%$ recommended fertilizer at $225-112-168 \mathrm{NPK} \mathrm{kg} \mathrm{ha}{ }^{-1}$ and $25 \%$ more than recommended fertilizer at 281.25-140-210 NPK kg $\mathrm{ha}^{-1}$. The mean values of these both NPK levels were statistically non-significant.

However, all inadequate applications beyond recommended fertilizer significantly recorded less value of all the agronomic traits. The minimum tillers plant $^{-1}(3.6),(4.2)$ plant height $(212.8 \mathrm{~cm})$, $(206.6 \mathrm{~cm})$ stem girth $(2.36 \mathrm{~cm}),(2.23 \mathrm{~cm})$, internodes plant $^{-1}$ (18.77), (18.11), millable canes (43.3 thousands ha $\left.{ }^{-1}\right),\left(42.8\right.$ thousands $\left.\mathrm{ha}^{-1}\right)$ and cane yield $\left(33.0 \mathrm{t} \mathrm{ha}^{-1}\right),\left(31.0 \mathrm{t} \mathrm{ha}^{-1}\right)$ plant and ratoon crop respectively were noted where no fertilizer was applied to the crop (Table 1). Inorganic NPK fertilizers applied based on some soil test recommendations have increasing effects on crop yields. However, inadequate and excessive applications of NPK fertilizers not only limit crop yield but may increase soil and environment problems [21].

Heavy doses of NPK fertilizers are not be economical to the farmer and would not be eco-friendly. 


\section{Effect of inorganic NPK on physiological traits of} plant and ratoon crop

The analysis of variances for physiological traits of sugarcane plant crop as affected by inorganic NPK levels showed significant difference at 5\% probability. The physiological traits of sugarcane plant and ratoon sugarcane as affected by inorganic NPK levels showed higher leaf area plant ${ }^{-1}$ $\left(5300-5400 \mathrm{~cm}^{2}\right),\left(5539-5643 \mathrm{~cm}^{2}\right)$, leaf area index (9.54-9.72), (9.97-2.16), crop growth rate (8.33-8.42 $\mathrm{gm}^{-2}$ day $\left.^{-1}\right),\left(9.75-9.85 \mathrm{gm}^{-2} \mathrm{day}^{-1}\right)$ and dry matter $\left(5000-5050 \mathrm{gm}^{-2}\right),\left(5225-5277 \mathrm{gm}^{-2}\right)$ respectively in plant and ratoon sugarcane crop with the application of $100 \%$ recommended fertilizer at 225-112-168 NPK $\mathrm{kg} \mathrm{ha}^{-1}$ and $25 \%$ more than recommended fertilizer at 281.25-140-210 NPK kg ha ${ }^{-1}$. The mean values of these both NPK levels were statistically non-significant.

However, all reduced applications beyond recommended NPK fertilizer significantly recorded lesser values of all the physiological traits of sugarcane sugarcane crop. The minimum leaf area plant $^{-1}\left(3000 \mathrm{~cm}^{2}\right),\left(2917 \mathrm{~cm}^{2}\right)$ leaf area index (5.40), (5.25) crop growth rate $\left(5.33 \mathrm{gm}^{-2} \mathrm{day}^{-1}\right),\left(5.63 \mathrm{gm}^{-2}\right.$ day $\left.^{-1}\right)$ and dry matter $\left(3200 \mathrm{gm}^{-2}\right),\left(3017 \mathrm{gm}^{-2}\right)$ in plant and ratoon sugarcane respectively, were observed in control plots where no fertilizer was applied (Table-2).

Effect of inorganic NPK on qualitative traits of plant and ratoon crop

All the qualitative traits of sugarcane plant crop as affected by inorganic NPK levels showed statistically significant difference at $5 \%$ probability level. The results of the study revealed maximum brix (22.63$22.83 \%)$, (23.16\%), pol (18.92-19.09\%), (19.22$19.37 \%)$, purity $(83.60-83.61 \%),(82.90-83.67 \%)$ and commercial cane sugar (13.66-13.77 \%), (13.79$13.98 \%$ ) with the application of $100 \%$ recommended fertilizer at 225-112-168 NPK kg ha ${ }^{-1}$ and $25 \%$ more than recommended fertilizer at 281.25-140-210 NPK $\mathrm{kg} \mathrm{ha}{ }^{-1}$ from plant and ratoon sugarcane, respectively. The mean values of both NPK levels were statistically non-significant. However, all inadequate and or excess applications beyond these doses significantly recorded less value of all the qualitative traits of sugarcane plant and ratoon suagrcane crop. The minimum brix (20.63\%), (20.80 $\%)$, pol (15.67\%),(15.87 \%), purity (75.95 $\%),(76.03)$ and commercial cane sugar (10.52\%), $(10.70 \%)$ were found in where no fertilizer was applied to the plant and ratoon sugarcane, respectively during growing season, except fiber $(12.28 \%)$, being higher in control plots where no fertilizer was applied and or in the plots where higher NPK fertilizer levels were applied (Table-3 ).

Effect of inorganic NPK on nutrient concentration and uptake in plant and ratoon crop

Statistical analysis of variances for nutrient (NPK) concentration and uptake in leaves of sugarcane plant crop as affected by inorganic NPK levels showed significant difference at $5 \%$ probability level. The results of the study showed maximum concentration of $\mathrm{N}(1.36 \%), \mathrm{P}(0.37 \%)$ and $\mathrm{K}(1.63 \%)$ with $\mathrm{N}$ uptake $\left(131.50 \mathrm{~kg} \mathrm{ha}^{-1}\right), \mathrm{P}$ uptake $\left(35.70 \mathrm{~kg} \mathrm{ha}^{-1}\right)$ and $\mathrm{K}$ uptake $\left(157.27 \mathrm{~kg} \mathrm{ha}^{-1}\right)$ in leaves with the application of $25 \%$ more fertilizer (281.25-140-210 NPK kg ha ${ }^{-1}$ ) than recommended dose, followed by application of $100 \%$ recommended NPK dose (225$112-168 \mathrm{~kg} \mathrm{ha}^{-1}$ ) which recorded $\mathrm{N}$ concentration of $1.35 \%, \mathrm{P} 0.30 \%$ and $\mathrm{K} 1.58 \%$ with $\mathrm{N}$ uptake of 128.50, $\mathrm{P}$ uptake 28.40 and $\mathrm{K}$ uptake $150.37 \mathrm{~kg} \mathrm{ha}^{-1}$ in foliage in plant crop. However, ratoon sugarcane shows somewhat different trend and the data exhibited that the maximum N P K concentration $(1.35-1.36 \%),(0.37-0.39 \%),(1.62-1.64 \%)$ and uptake of $129.77-131.30 \mathrm{~kg} \mathrm{ha}^{-1}, 35.20-38.23 \mathrm{~kg} \mathrm{ha}^{-1}$ and $154.67-160.17 \mathrm{~kg} \mathrm{ha}^{-1}$, respectively with the application of $100 \%$ recommended fertilizer at 225 112-168 NPK kg ha ${ }^{-1}$ and $25 \%$ more than recommended fertilizer at 281.25-140-210 NPK kg $\mathrm{ha}^{-1}$ respectively. All the remaining fertilizer treatments recorded lower values of NPK concentration and uptake in leaves. The minimum leaf NPK concentration $(1.14,0.19$ and $1.21 \%$ respectively) and NPK uptake (61.23, 10.46 and $64.67 \mathrm{~kg} \mathrm{ha}^{-1}$ respectively) were observed in unfertilized plots (Table-4).

\section{Conclusion}

In this study the all the agronomic, physiological, quality and nutrient uptake traits in plant and ratoon sugarcane crops were proved higher with the application of $100 \%$ recommended fertilizer at 225 112-168 NPK kg ha ${ }^{-1}$ and $25 \%$ more than recommended fertilizer at $281.25-140-210 \mathrm{NPK} \mathrm{kg}$ $\mathrm{ha}^{-1}$. The mean values of these both NPK levels were statistically non-significant. However, all inadequate applications beyond recommended applications significantly recorded less value of all traits of sugarcane plant and ratoon crop. Further increase in NPK rates showed non-significant differences for these traits. However, inadequate and excessive applications of NPK fertilizers are neither economical for the farmer and nor eco-friendly, hence the fertilizer at 225-112-168 NPK kg ha ${ }^{-1}$ is best suited for getting optimum sugarcane yield from the variety Thatta- 10 . 


\section{References}

1. Khan, I. A., M. A. Javed, A. Khatri, M. A. Siddiqui, M. K. R. Khan, N. A. Dahar, M. H. Khanzada and R. Khan (2002). Performance of exotic sugarcane clones at NIA, Tando Jam. Asian J. Plant Sci. 1: 238-240.

2. Ali, C.K., M.A. Nadeem, and M.A. Javid (1997). Effect of nitrogen levels and $\mathrm{P}$ application strategy on sunflower. Fertiliser News. 42 (3):43-47.

3. Korndorfer, G. H. (1990). Potassium and sugarcane quality. Informacoes Agrono. 49: 1-3.

4. Miller J. D. and R. A. Gilbert (2006). Florida Sugarcane Handbook This document is SS-AGR234, one of a series of the Agronomy Department, Florida Cooperative Extension Service, Institute of Food and Agric. Sci. Univ. of Florida.

5. Khan, I. A., A. Khatri, G. S. Nizamani, M. A. Siddiqui, S. Raza and N. A. Dahar (2005). Effect of NPK fertilizers on the growth of sugarcane clone AEC86-347 developed at NIA, Tando Jam, Pakistan. Pak. J. Bot. 37 (2): 355-360.

6. Mahar, G.M., U. A. Buriro, F. C. Oad and.S. A. Shaikh (2008). Cane Yield and Sugar Recovery of Sugarcane Variety Larkana-2001under Different Fertilizer Sources. Asian J. Plant Sci. 7 (2): 237-240.

7. Milford, G. F. J., M. J. Armstrong, P. J. Jarvis, B. J. Houghton, D. M. Bellett-Travers, J. Jones and R. A. Leigh (2000). Effects of potassium fertilizer on the yield, quality and potassium off take of sugar beet crops grown on soils of different potassium status. J. Agric. Sci. 135: 1-10.

8. Wood, R. A. (1990). The roles of nitrogen, phosphorus and potassium in the production of sugarcane in South Africa. Fertilizer Res. 26: 8798.

9. Malik, N. A., S. Afghan, I. Ahmed and R. A. Mahmood (1993). Response of sugar cane to different doses of NPK fertilizer in Somalia. Pak. Sugar J. 7: 7-9.

10. Rabindra, S., S. N. Swamygowda and T. G. Devi (1993). Long term effect of fertilizers on sugarcane. Current Res. 22: 6-8.
11. Sharif, M. and T. M. Chaudhry (1988). Role of Potash and profitability of NPK applications to sugarcane under Sindh conditions. In: Proc. $1^{\text {st }}$ Natl.Congr. Soil Sci. Lahore, Oct. pp. 327-333.

12. Khan, I. A., M. A. Javed, A. Khatri, M. A. Siddiqui, M. K. R. Khan, N. A. Dahar, M. H. Khanzada and R. Khan (2002). Performance of exotic sugarcane clones at NIA, Tando Jam. Asian J. Plant Sci. 1: 238-240.

13. Malik, K. B. (1990). Sugarcane production problems and research strategies for improvement. Dept. of Agric. Punjab.

14. Karstens, S., M. Ross, P. Luedders and A. Krauss 1992. Nutritive status of sugarcane in Punjab, Pakistan. Pak. J. Agric. Res. 13 (4): 327-333.

15. Bokhtiar, S. M. (2004). Scientific basis and scope of improvement of low sugarcane yield and recoverable sucrose in nutritional aspect of Bangladesh. Pak Sugar J. XIX (I): 27-36.

16. Raghaviah, C .V. and P. P. Singh (1980). Yield and nitrogen uptake of sugarcane varieties at graded levels of N. Indian Sugar. 29 (1): 13-17.

17. Trivedi, N. and S. K. Saini (1986). Response of sugarcane varieties on $\mathrm{N}$ application under Taral condition of Uttar Pradesh. Indian Sugar. 35 (11): 606-607.

18. Effect of planting dates, varieties and mineral nutrition on juice quality and cane productivity. Cooperative Sugar. 34 (100): 793-795.

19. Khan, I. A., A. Khatri, M. Ahmad, K. A. Siddiqui, N. A. Dahar, M. H. Khanzada and G. S. Nizamani (1997).Genetic superiority of exotic clones over indigenous clones for quantitative and qualitative traits. The Nucleus. 34: 153-156.

20. Ahmed, A. (1994). Sucrose-linked prices of cane to raise sugar export prospects, Agriculture and Industry, DAWN Economic and Business Review, July 9-15, III.

21. FAO (1999). Soil fertility initiative for SubSaharan Africa. World Soil Resource Reports. 85: 82. 